\title{
EMISSION REGIONS ON THE FLARE STAR EV Lac IN 1989
}

\author{
L.A.PUSTIL'NIK and I.P.PANFEROVA \\ Special Astrophysical Observatory Russian Acad. of Sci. \\ Niznij Arhyz, Stavropol Territory, 357147, Russia. \\ and \\ R.E.GERSHBERG and N.I.SHAKHOVSKAYA \\ Crimea Astrophysical Observatory, Crimea, Ukraine
}

\begin{abstract}
Emission spectra of the flare star EV Lac in several nights of September 1989 are studied. Their variations due to the stellar rotational modulation are found and permit to define the most active longitudes on the stellar surface.
\end{abstract}

Key words: activity-flares

\section{Introduction}

The activity of red dwarfs (flare stars of the UV Cet type) is determined by the dynamo processes generating the magnetic fields and their subsequent dissipation in the star's atmosphere. The direct measurements of such fields, which began recently, led to the conclusion that at strength of several kilogauss they occupy tens of percents of the active star's surface (Saar 1991). However, such direct measurements are extremely difficult, and the spectral and photometric observations of magnetism manifestation remain the most important way of the study of dynamo processes on such stars. The observers attention is traditionally concentrated on the flares in optic continuum (white flares) - the most expressive sings of dissipation of magnetic field energy. In our paper we tried to investigate a less violent but not less informative manifestation of the process, namely, an anomalous heating of the atmosphere over an active region, displaying itself in the dynamics of the emission spectra of these stars at time scale of minutes to days. Besides, the link of the zone of active regions concentration to a definite phase of the rotation period (active latitude) and its secular drift in time are also of great importance for the understanding of dynamo processes going on in the stellar convective zone.

\section{Observations}

For the consideration of this problem we carried out observations of the star EV Lac at the 6-meter telescope in the period of its cooperative investigations of 1989 (Berdyugin et al. 1992). The photon flow coming from the star was divided by a light divisor onto 2 devices: in a spectrograph SP-124 with 1000 channel television scanner and in a speed electrophotometer. The spectra obtained on the strobes of the television camera with a resolution of 3-6 $\AA$ were registered with a time resolution which was chosen from 30 to $300 \mathrm{sec}$. The dead time between spectral exposures needed for the record of an accumulated spectrum to magnetic disc was $15 \mathrm{sec}$. Due to this methodic we had a possibility to carry out synchron observations of brightness variation with high temporal resolution and to register simultaneously the spectral variations at times of the order of minutes. 


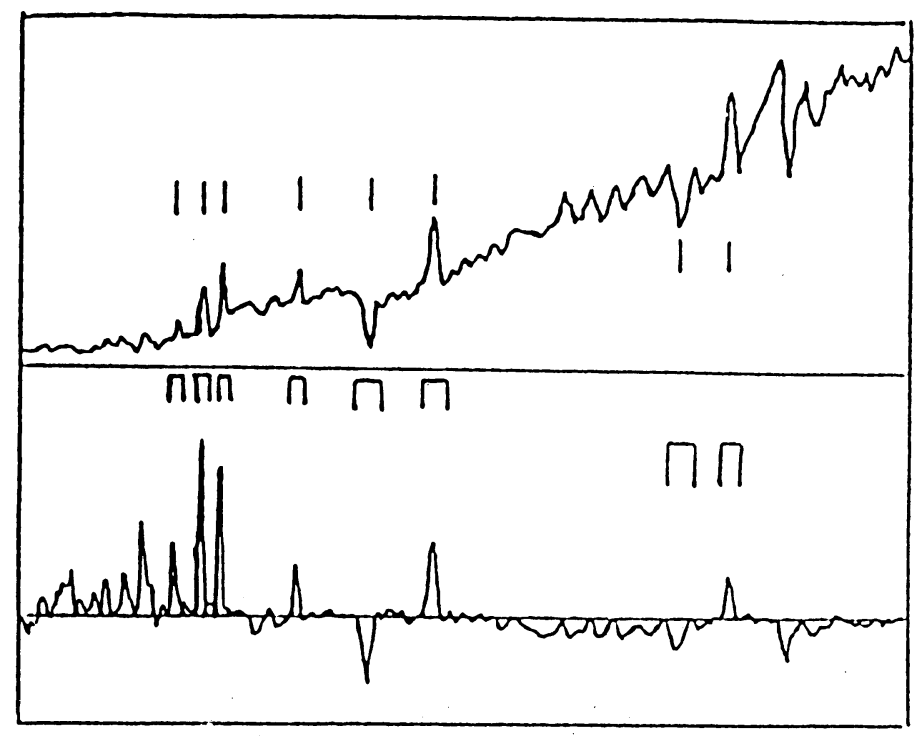

Fig. 1. The average spectrum of EV Lac and the spectrum reduced to quasi continuum

For all that the community of coming photon flow defends against difficulties connected with local effects of atmosphere, fibers and system of registration inherent to observations at independent removed devices. During observations more or less prominent flares of EV Lac were not detected. That is why the analysis of the obtained spectral data was turned to the search of star spectrum changes which are not connected with flares, and to the search of weak flares effects, which were not registered individually. First, every obtained spectrum was reduced for unhomogeneous sensibility of cathode and spectral sensibility of scanner. Then with the help of the record of an argon-neon lamp spectrum a homogeneous link of every spectrum to wave length was carried out. All so reduced individual spectra were summed up and gave an average star spectrum out of flare. On this sum spectrum with low noise the regions out of prominent emission and absorption lines were selected and through these regions with the help of spline curves a quasi continuum of the stellar radiation was drawn.

In Fig. 1 there are an initial average spectrum of EV Lac and the spectrum reduced for so drawn continuum. In the figure all spectral details are marked which undergone the following quantity analysis in the course of which we determined equivalent widths of emission lines $\mathrm{H}_{\beta}, \mathrm{H}_{\gamma}, \mathrm{H}_{\delta}, \mathrm{H}_{\epsilon}+\mathrm{HCaII}, \mathrm{KCaII}$ and $\mathrm{H}_{8}$ and absorption lines $\lambda 4227 \AA \mathrm{CaI}, \lambda 4780 \AA \mathrm{AMgH}$, the jump of intensity near the head of the molecular band $\mathrm{TiO} \lambda 4955 \AA$ and flow intensity in several emission lines. Using the quasi continuum regions selected in the sum spectrum, we carried out 
TABLE I

Equivalent widths and ratio of fluxes of lines.

\begin{tabular}{llllll}
\hline Average spectrum of $\ldots$ & $3-6.9$. & $3 / 4.9$. & $4 / 5.9$. & $5 / 6.9$. & $6 / 7.9$. \\
\hline & & & & & \\
Sum exposure (min) & 237 & 60 & 78 & 47 & 52 \\
The number of spectra & 49 & 20 & 12 & 8 & 9 \\
$W_{H_{\beta}}(\AA)$ & 5.2 & 4.9 & 6.3 & 6.0 & 3.3 \\
$W_{H_{\gamma}}(\AA)$ & 9.9 & 9.3 & 10.4 & 12.7 & 8.2 \\
$W_{H_{\delta}}(\AA)$ & 5.0 & 6.4 & 6.8 & 3.7 & 2.5 \\
$W_{H_{\epsilon}+H C a I I}(\AA)$ & 12.7 & 11.4 & 15.6 & 11.5 & 10.9 \\
$W_{K C a I I}(\AA)$ & 15.7 & 14.4 & 19.1 & 16.2 & 12.2 \\
$W_{H_{8}}(\AA)$ & 5.6 & 6.4 & 6.3 & 6.8 & 3.7 \\
$W_{\lambda 4227 C a I}(\AA)$ & 15.1 & 16.9 & 14.4 & 15.5 & 14.1 \\
$W_{\lambda 4780 M g H}(\mathrm{AA})$ & 7.1 & 9.0 & 6.1 & 7.9 & 5.4 \\
$I_{\lambda-4955} / I_{\lambda}+4955$ & 1.6 & 1.7 & 1.51 & 1.6 & 1.6 \\
$F_{H_{\gamma}} / F_{H_{\beta}}$ & 0.82 & 0.81 & 0.69 & 0.84 & 1.26 \\
$F_{H_{\delta}} / F_{H_{\beta}}$ & 0.32 & 0.38 & 0.34 & 0.21 & 0.35 \\
$F_{H_{\epsilon}+H C a I} / F_{H_{\beta}}$ & 0.53 & 0.45 & 0.52 & 0.41 & 0.92 \\
$F_{K C a I} / F_{H_{\epsilon}+H C a I}$ & 0.88 & 0.86 & 0.88 & 0.95 & 0.83 \\
\hline
\end{tabular}

the indicated procedure with every individual record of spectrum, with sum spectra of every of 4 nights and with the sum spectrum of all nights.

\section{Discussion}

Table 1 allows us to suspect valuable changes of the emission star spectrum from night to night. Really, whereas the jump value near the limit of molecular band $\lambda 4955 \AA \mathrm{TiO}$ and the equivalent width of absorption line $\lambda 4227 \AA \mathrm{CaI}$ are practically constant, i.e. the spectrum of photosphere radiation does not change, the chromospheric emission lines $H_{\beta}$ and $H_{\gamma}$ in the first the fourth nights were obviously weaker than in two nights between them. Allowing for the fact that the rotation period of EV Lac is 4.38 days (Roizman 1984), it is naturally to consider that on the 3d and 6th of September the star was turned to the Earth with the hemisphere less covered by active regions than on the 4th and 5 th of Sept. An analogous modulation of chromospheric radiation of $\mathrm{EV}$ Lac was discovered in Crimea observations of 1986 and the maximum of chromospheric emission in the line $H_{\alpha}$ corresponded to the minimum of photospheric radiation which was stipulated by the turn of most spotted hemisphere of star towards the Earth (Gershberg et al. 1991). It can be considered that up to half of day the maximum of chromospheric radiation of EV Lac took place between our observations on the $4 / 5$ and $5 / 6$ of September 1989, i.e. J.D. 244775,0 \pm 0,5. If using Roizman's ephemerids (Roizman 1984), this moment corresponds to the phase $0.6 \pm 0.1$.

On the other hand, the purely photometric observations of EV Lac in 1986, 1987 and 1990 led to the conclusion that the phases of maximum spottedness of 


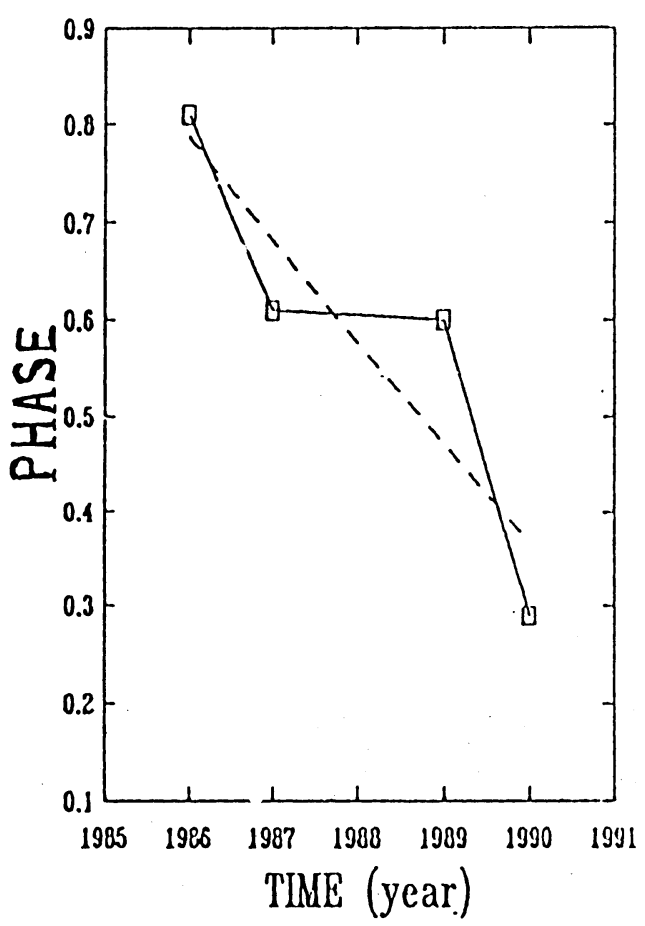

Fig. 2. Time evolution of phase the most spotted photospheric region.

the star in these years are the following: $0.81,0.61,0.29$.

The common consideration of these data reveals a systematic movement, whose linear part can be stipulated by the error of the adopted period, and deviations from the linear character can be stipulated by a real drift of the most spotted photospheric region along the star's surface (Fig.2).

The analysis of the spectra dynamics during the night allows us to suspect the presence of emission lines activity during relatively quiet and flareless periods of observations (see Fig.3). 


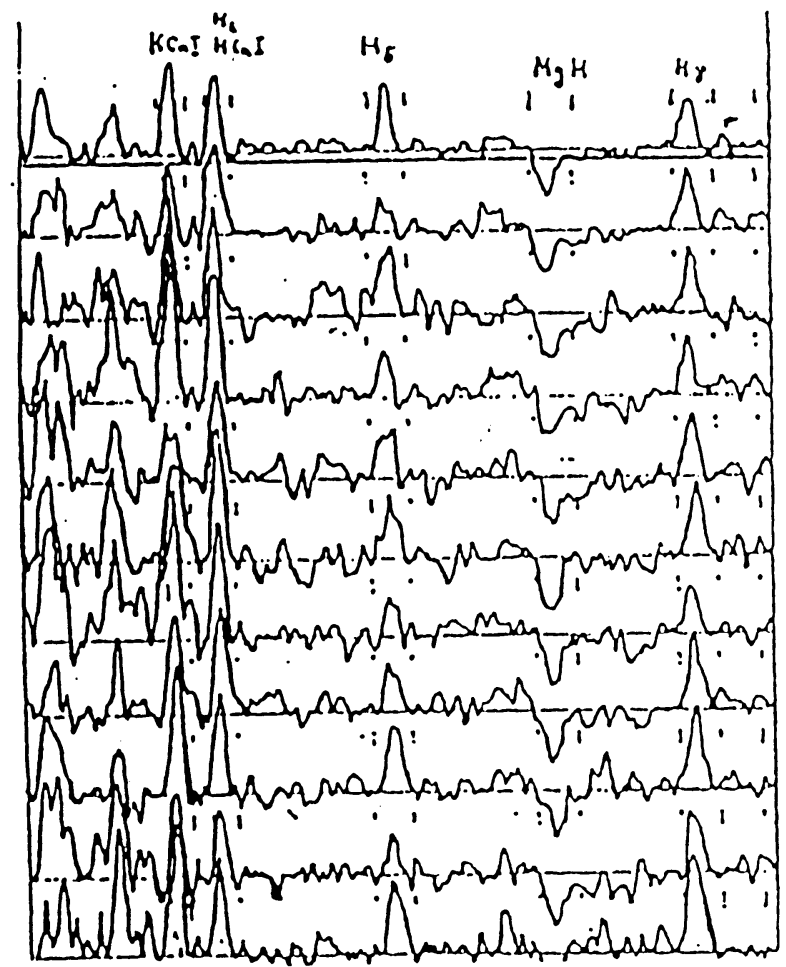

Fig. 3. The dynamics of reduced spectra of EV Lacv on the time scale 300 sec.

Though, for more confident conclusions a further analysis of possible randomizing factors influencing the characteristics of observed lines in necessary.

\section{References}

Saar, S.H.:1991, in I. Tuominen, D. Moss, G. Ruediger (eds) “The Sun and Cool stars: activity, magnetism, dynamos", Lectures Notes in Physics 380, 389.

Berdyugin, A.V., Gershberg, R.E., Malanushenko, V.P., Shahovskaya, N.I., Shahovskoy, N.M., Garbusov, G.A., Pagano, I., Pustil'nik, L.A., Panferova, I.: 1992, Isvestia Kricmskoy Astrophys. Observatorii 89, in print.

Gershberg, R.E., Ilyin, I.V., Shahovskayia, N.I.: 1991, Astron. Zh. 68, 959.

Roizman, G.Sh.: 1984, Pis'ma v Astron. Zh. 10, No. 5, 279. 